\title{
The Upgrade of the ATLAS Electron and Photon Triggers towards LHC Run 2 and their Performance
}

\section{Sebastien Kahn* on behalf of the ATLAS collaboration}

Centre de Physique des Particules de Marseille (CPPM), Aix-Marseille Université, CNRS/IN2P3

E-mail: kahnecppm.in2p3.fr

\begin{abstract}
Electron and photon triggers covering transverse energies from $5 \mathrm{GeV}$ to several $\mathrm{TeV}$ are essential for signal selection in a wide variety of ATLAS physics analyses to study Standard Model processes and to search for new phenomena. Final states including leptons and photons had, for example, an important role in the discovery and measurement of the Higgs particle. Dedicated triggers are also used to collect data for calibration, efficiency and fake rate measurements. The ATLAS trigger system is divided in a hardware-based and a software based high level trigger, both of which were upgraded during the long shutdown of the LHC in preparation for data taking in 2015. The increasing luminosity and more challenging pile-up conditions as well as the planned higher center-of-mass energy demanded the optimisation of the trigger selections at each level to control the rates and keep efficiencies high. The evolution of the ATLAS electron and photon triggers and their performance will be presented, including initial results from the early days of the LHC Run 2 operation.
\end{abstract}

The European Physical Society Conference on High Energy Physics

22-29 July 2015

Vienna, Austria

${ }^{*}$ Speaker. 


\section{Introduction}

Many physics analyses in the ATLAS experiment [1] at the LHC involve electrons or photons in their final state. Those objects were widely used for the discovery of the Higgs boson [2] , searches for beyond Standard Model processes and precision measurements of Standard Model processes. Between 2012 (end of Run 1) and 2015 (beginning of Run 2) the centre-of-mass energy of the proton-proton collisions has increased from $8 \mathrm{TeV}$ to $13 \mathrm{TeV}$, long-term average instantaneous luminosity is expected to increase from $8 \times 10^{33} \mathrm{~cm}^{-2} \mathrm{~s}^{-1}$ to $1.6 \times 10^{34} \mathrm{~cm}^{-2} \mathrm{~s}^{-1}$ and proton bunch spacing will be reduced from $50 \mathrm{~ns}$ to $25 \mathrm{~ns}$. This is expected to induce a factor 4-5 increase of the electron-photon trigger rate. This document describes the upgrade of the Run 2 electron and photon triggers and their performance measured with early Run 2 data.

\section{Electron and photon trigger algorithms}

The selection of events with electrons or photons was performed in two steps in Run 1. The first step also referred as Level 1 (L1), is using fast custom hardware that triggers on the data using the calorimeter information. The second step, the High Level Trigger (HLT) uses software criteria to provide additional rejection of the hadrons reconstructed as electrons or photons using all the information provided by the calorimeter and tracking detectors.

Energy clusters in the electromagnetic calorimeter are reconstructed with a sliding window algorithm with a coarse granularity. The L1 trigger items are then defined with an energy threshold of the corresponding energy clusters and further electromagnetic and hadronic isolation requirements. At the HLT, tracks are reconstructed in a region of interest around the energy cluster, and matched to them. If a relevant track is found, the energy cluster will be used to define an electron trigger and if no track is matched the cluster will be used to define a photon trigger.

The HLT photon triggers are defined using a sequence of cuts (cut-based) on relevant identification variables. In the electron case, two different kinds of triggers are defined using either cut-based identification criteria or the probability density function of relevant electron identification variables (likelihood). Shower shape identification variables using the full granularity of the calorimeters are used to reduce the trigger rate arising from hadrons reconstructed as electrons or photons. For the electron triggers, track quality, track isolation and track-cluster matching identification variables are also used to further reduce the rate. The information from transition radiation arising in the outermost part of the Inner detector, the Transition Radiation Tracker, is also used to define the final electron triggers. The defined trigger requirements depend on the computed transverse energy $\left(E_{\mathrm{T}}\right)$ and the pseudorapidity $\left(\eta^{1}\right)$ associated to the reconstructed electron/photon.

\section{Upgrade of the electron and photon triggers for Run 2}

The increase of the center-of-mass energy collision from $7 \mathrm{TeV}$ to $13 \mathrm{TeV}$ and the luminosity increase are expected to multiply the trigger rates by a factor 4-5. Therefore, the main constraint for

\footnotetext{
${ }^{1}$ The ATLAS coordinate system is defined such that the $\mathrm{x}$-axis points towards the center of the LHC ring, the y-axis points up, the z- axis defines a right-handed coordinate system and $r=\sqrt{x^{2}+y^{2}} \cdot \phi$ is the azimuthal angle defined such that $\phi=0$ is along the $\mathrm{x}$-axis. The pseudorapidity is $\eta=\ln \tan (\eta / 2)$, where $\theta$ is the polar angle measured from the beam pipe.
} 
the definition of the trigger items was to fit in the available trigger bandwidth keeping the transverse energy threshold as low as possible and the efficiency as high as possible. To achieve this goal, both L1 and HLT triggers have been upgraded.

\subsection{L1 trigger upgrades}

The global L1 trigger bandwidth has been increased from $70 \mathrm{kHz}$ to $100 \mathrm{kHz}$ with respect to Run 1 thanks to new hardware, firmware and connection between the L1 trigger modules. The event topology will be used in the near future to define triggers dedicated to specific final states at L1.

The calorimeter energy computation is now improved thanks to a new hardware module (New Multi Chip Module) that allows a better pile-up and noise subtraction using autocorrelation filtering. The processor dedicated to the cluster reconstruction has also been updated and allows now to use $E_{\mathrm{T}}$ dependent isolation criteria (electromagnetic or hadronic) with a $\Delta E_{\mathrm{T}} \sim 0.5 \mathrm{GeV}$ energy resolution. The module that merges the triggers defined with calorimeter information has been extended. Now 16 different $E_{\mathrm{T}}$ threshold can be defined (8 in Run 1) with a $\Delta \eta=0.1$ pseudorapidity granularity.

\subsection{HLT trigger upgrades}

In Run 1 the HLT selection was processed in two steps. In the first step called Level 2 (L2), a first selection of the event was performed using simplified reconstruction and identification criteria. In the second step called the Event Filter (EF) a complete object reconstruction was performed and the final identification criteria were defined using the best precision available online.

The two step HLT selections have been merged for Run 2, making it is possible to perform a fast track/calorimeter reconstruction step, select the events of interest and then use the already computed information for a precision track/calorimeter reconstruction for the final HLT trigger requirements. Additional computing resources have also been added. Following these and many other upgrade, the available HLT bandwidth increased from $600 \mathrm{~Hz}$ to $1000 \mathrm{~Hz}$.

The clustering and tracking algorithms used for the HLT trigger have been improved and are now closer to offline. The energy calibration is now based on a multivariate analysis. Moreover, the identification criteria used for the electron and photon triggers have been optimized for Run 2 conditions. The electron trigger is now based on a likelihood technique (cut-based in Run 1) expected to achieve a rejection two times higher while maintaining the same efficiency. Cut-based electron triggers have also been optimized for the commissioning of the likelihood triggers on early data.

\section{Trigger performances}

\subsection{Electron trigger}

To make the most of the available data, the $E_{\mathrm{T}}$ thresholds of the primary (un-prescaled) single electron triggers are kept as low as possible and adapted to the luminosity of the data taking. The $E_{\mathrm{T}}$ thresholds will then increase from 24 to $26-28 \mathrm{GeV}$ in correspondence with the increase of the instantaneous luminosity. For the same reason, the identification criteria will be tightened with the luminosity increase. An additional loose requirement on the relative track isolation within a 
cone of $R=0.2$ is applied, $p_{T}^{i s o} / E_{\mathrm{T}}<0.1$. A primary di-electron trigger is also defined using two electrons with a $17 \mathrm{GeV} E_{\mathrm{T}}$ threshold and loose identification criteria. Other combined triggers are defined using for example an electron and muon object and a lower energy threshold.

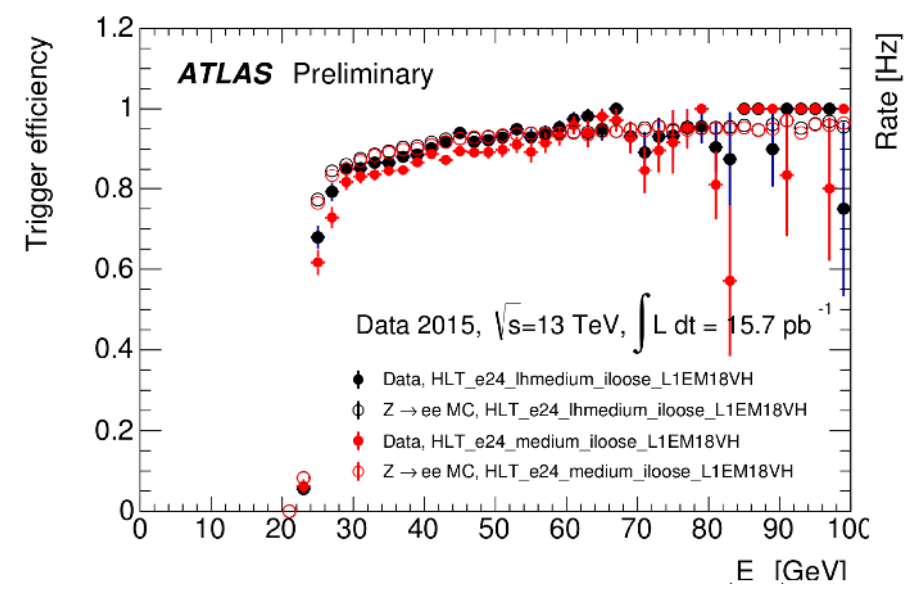

Figure 1: Efficiencies of the primary single electron triggers as a function of the offline $E_{\mathrm{T}}$. The open points corresponds to the efficiencies measured using a $Z \rightarrow e e$ Monte Carlo simulation, and the solid ones to efficiency measured on data using a $Z$ tag-and-probe method. The red points correspond to the triggers using cut-based identification criteria and the black ones using likelihood criteria. Taken form Ref.[5].

Figure 1 [5] shows the $E_{\mathrm{T}}$ dependence of the efficiency of the primary single electron trigger defined with $24 \mathrm{GeV} E_{\mathrm{T}}$ threshold, medium identification requirements and loose requirement on the relative track isolation within a cone of $R=0.2$ is applied, $p_{T}^{i s o} / E_{\mathrm{T}}<0.1$ computed with simulated and real data. The efficiencies were measured with a tag-and-probe method using $Z \rightarrow e e$ decays [3] with no background subtraction applied. They are compared to the expectation from $Z \rightarrow e e$ simulation. An efficiency of $80 \%$ is reached at $26 \mathrm{GeV}$ for the simulation and at $30 \mathrm{GeV}$ for both cut-based and likelihood based triggers. Then the efficiency slowly increases from $80 \%$ to $90 \%$ for both cut-based and likelihood triggers. The inefficiency in data primarily arises at the last step of the HLT selection that requires tracking related and track-cluster matching criteria. The cut-based trigger efficiency measured on data is $\sim 4 \%$ lower on data than in the simulations whereas the data to Monte Carlo discrepancy is $1-2 \%$ for the likelihood triggers depending on the considered $E_{\mathrm{T}}$ range.

Figure 2 [5] shows the trigger rate associated to the primary single electron triggers measured on data as a function of the instantaneous luminosity. The rate of the triggers using a medium identification criteria are $\sim 1.5 \mathrm{~Hz}$ for an instantaneous luminosity of $1 \times 10^{32} \mathrm{~cm}^{-2} \mathrm{~s}^{-1}$ and $\sim 1$ $\mathrm{Hz}$ for the triggers using a tight identification. The likelihood trigger rates are on average $25 \%$ lower than the cut-based ones for a $6 \%$ better electron efficiency. The trigger rates are linear as a function of the instantaneous luminosity.

\subsection{Photon trigger}

Primary single photon triggers are defined using loose cut-based identification criteria with a 120-140 GeV $E_{\mathrm{T}}$ threshold. The primary di-photon trigger are defined using two photons with a 25 


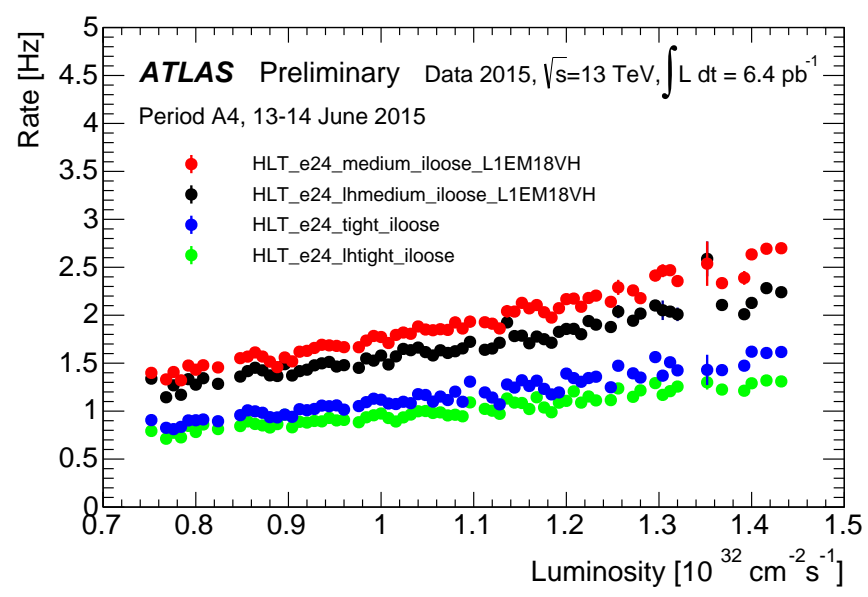

Figure 2: Output rates of single electron triggers as a function of the instantaneous luminosity during the 2015 proton-proton data taking at a center-of-mass energy of $13 \mathrm{TeV}$ and a LHC bunch-crossing interval of 50 ns. Taken form Ref.[5].

(35) $\mathrm{GeV} E_{\mathrm{T}}$. A loose [4] identification criteria will be first used and a medium [4] one for higher luminosity.

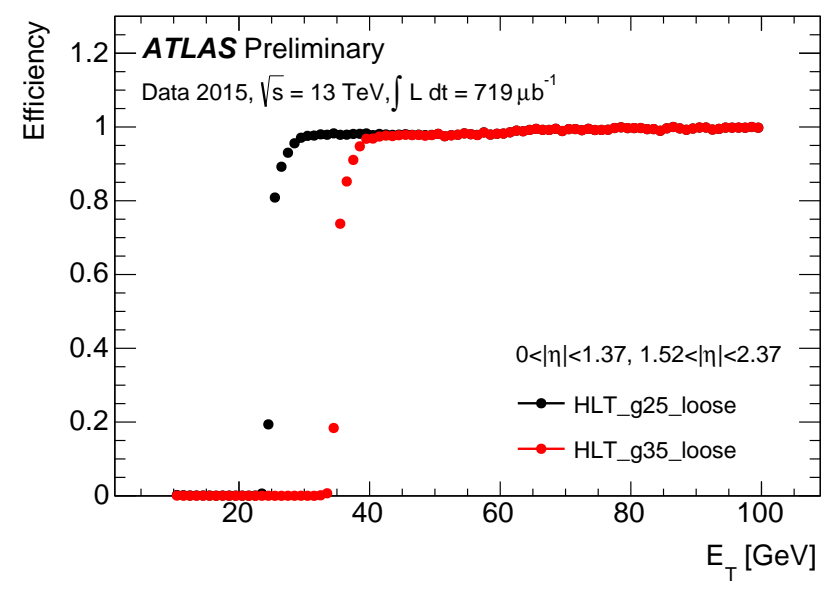

Figure 3: Efficiency of photon triggers items used to define the di-photon triggers requiring a $E_{\mathrm{T}}>25 \mathrm{GeV}$ (black circles) and $E_{\mathrm{T}}>35 \mathrm{GeV}$ (red circles) and loose photon identification criteria with respect to photon candidates reconstructed offline passing the tight identification selection as a function of the offline photon transverse energy for $|\eta|<2.37$ excluding the transition region between the barrel and endcap electromagnetic calorimeters at $1.37<|\eta|<1.52$. The efficiency is measured using events recorded with a level-1 trigger requiring an electromagnetic cluster with $E_{\mathrm{T}}>7 \mathrm{GeV}$. No background subtraction is applied. The error bars represent the statistical uncertainty which is calculated using a Bayesian estimate with Jeffrey's prior. Taken form Ref.[5].

Figure 3 [5] shows the $E_{\mathrm{T}}$ dependence of the trigger items used to define the primary di-photon trigger. A plateau with an efficiency larger than $99 \%$ is reached at $30(40) \mathrm{GeV}$ for the trigger item 
defined with a $25(35) \mathrm{GeV}$ threshold.

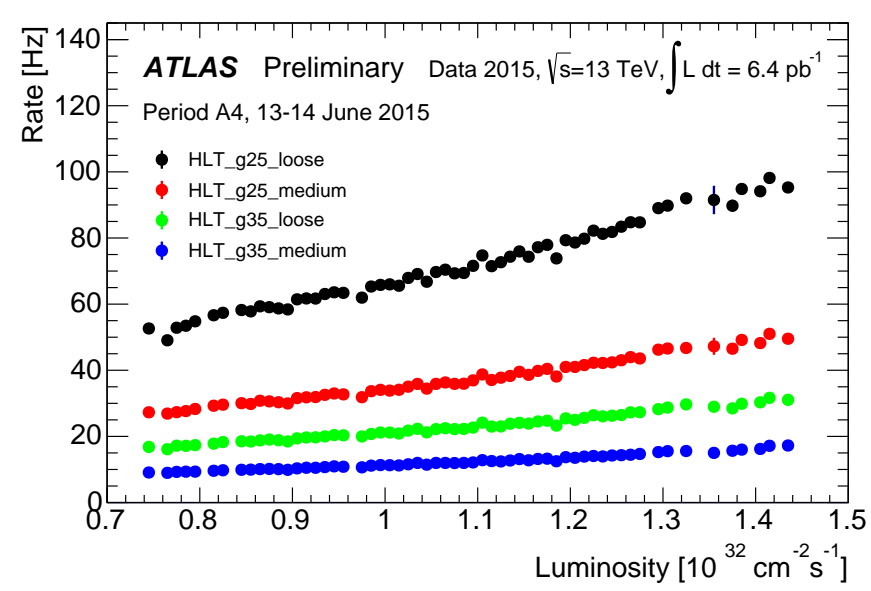

Figure 4: Output rates of photon triggers items used to define the di-photon triggers as a function of the instantaneous luminosity during the 2015 proton-proton data taking at a center-of-mass energy of $13 \mathrm{TeV}$ and a LHC bunch-crossing interval of $50 \mathrm{~ns}$. The triggers require a $E_{\mathrm{T}}$ threshold of $25 \mathrm{GeV}$ or $35 \mathrm{GeV}$ and either cut-based loose or medium identification. They also apply at the level-1 an $E_{\mathrm{T}}$ dependent veto on the energy deposited in the hadronic calorimeter behind the electromagnetic energy cluster. Taken form Ref.[5].

Figure 4 [5] shows the trigger rate associated to the trigger items used to define the di-photon triggers as a function of the instantaneous luminosity. The rates associated to the trigger items defined with a $25 \mathrm{GeV} E_{\mathrm{T}}$ threshold are $\sim 3$ times larger than the ones defined with a $35 \mathrm{GeV} E_{\mathrm{T}}$ threshold. The rate associated to the trigger items defined with a loose identification criteria are twice as high as the ones defined with a medium identification criteria. The trigger rates are linear as a function of the instantaneous luminosity.

\section{Conclusions}

Good performances of the electron and photon triggers has been measured on data in Run 1 . To handle the factor 4-5 increase in trigger rate expected between Run 1 and Run 2, both the electron and photon triggers optimized for Run 2 conditions in both hardware and software side. Good performances have been measured using early Run 2 data.

\section{References}

[1] ATLAS collaboration, The ATLAS Experiment at the CERN Large Hadron Collider, JINST 3 (2008) S08003.

[2] ATLAS collaboration, Observation of a new particle in the search for the Standard Model Higgs boson with the ATLAS detector at the LHC, Phys. Lett. B 716, 1âĂŞ29 (2012) .

[3] ATLAS collaboration, Electron efficiency measurements with the ATLAS detector using the 2012 LHC proton-proton collision data (2014), ATLAS-CONF-2014-032, https://cds.cern.ch/record/1706245. 
[4] ATLAS collaboration, Measurements of the photon identification efficiency with the ATLAS detector using 4.9 fbấL Šl of pp collision data collected in 2011 (2012), ATLAS-CONF-2012-123, https://cds.cern.ch/record/1473426.

[5] ATLAS collaboration, Public Egamma Trigger Plots for Collision Data, https://twiki.cern.ch/twiki/bin/view/AtlasPublic/EgammaTriggerPublicResults.

[6] ATLAS Collaboration, Performance of the ATLAS Electron and Photon Trigger in p-p Collisions at sqrts $=7 \mathrm{TeV}$ in 2011, ATLAS-CONF-2012-048, https://cds.cern.ch/record/1450089.

[7] Moritz Backes, The ATLAS Trigger System Ready for Run-2, these proceedings. 